\title{
Influence of Stretching and Strengthening of the Iliopsoas Associated with Lumbar Segmental Stabilization Exercises in Patients with Low Back Pain: The pilot study
}

\author{
Claudia Puzzoni Volpatoํㅜ, Marco Aurélio Nemitalla Added ${ }^{2 *}$, Graziella Candido de Paula X. Richter², \\ Valter Tanaka ${ }^{2}$, Nilza Aparecida Almeida de Carvalho ${ }^{3}$ and Diego Galace de Freitas ${ }^{3}$ \\ ${ }^{1}$ Staff Physical Therapist, Irmandade da Santa Casa de Misericórdia de São Paulo, Rehabilitation Service, São Paulo-SP, Brazil \\ ${ }_{2}^{2}$ Master of Science student, Staff Physical Therapist, Irmandade da Santa Casa de Misericórdia de São Paulo, Rehabilitation Service, São Paulo-SP, Brazil \\ ${ }^{3}$ Associate Professor and Physical Therapist Staff, Irmandade da Santa Casa de Misericórdia, Physical Therapy Department, São Paulo-SP, Brazil
}

Received: April 10, 2014; Accepted: May 22, 2014; Published: May 25, 2014

*Corresponding author: Marco Aurélio Nemitalla Added, Physical Therapy Department, Rua Curuzu no 314, Alto da Lapa, Sao Paulo - SP, Brazil, CEP: 05084-020; E-mail: marcoadded@terra.com.br

\begin{abstract}
Low back pain (LBP) is one of the principal causes of incapacity. One treatment approach for this pathology involves segmental stabilization. The iliopsoas muscle is also considered a potential stabilizer of the lumbar spine.

Objective: To compare the effects of stretching and strengthening of the iliopsoas muscle associated with segmental stabilization versus stabilization alone, in the treatment for LBP.

Methods: Fourteen patients were randomized into three groups. The first group was given only segmental stabilization training (ST); the second was submitted to ST and stretching of the iliopsoas muscle (ST-Stretch), and the third was given ST and strengthening of the iliopsoas (ST-Strength).
\end{abstract}

Results: The group ST $(p=0.006)$ and ST-Strength $(p=0.092)$ showed significant improvement in the VAS scale. The extension flexibility showed statistically significant improvement in the group ST- strength group $(\mathrm{p}=0.038)$.

Conclusion: ST alone, or associated with strengthening of the iliopsoas, proved more effective for improving lumbar pain and flexibility compared to ST Stretch.

Keywords: Low Back Pain; Lumbar Spine; Stability; Psoas Muscle

\section{Introduction}

Low back pain (LBP) is one of the leading causes of incapacity [1-3] and the high cost of treatment renders preventative strategies paramount [4] .

Chronic LBP is known to occur in the setting of reduced vertebral column stability, due to a loss of mechanisms of passive control (vertebras, discs and ligaments), active control (muscles and tendons) and of motor control $[5,6]$. The system of local stabilization involves deep intrinsic muscles which are directly attached to the lumbar vertebrae, and the global system comprises the great superficial muscles originating in the pelvis which insert in the thoracic cavity[4-6], with both systems necessary for stability and control of movements [7] .

Evidence suggests that the structure and function of the truncal deep muscles are altered in individuals with LBP[1,8$12,35]$. This has led to increased focus on exercises which aim to maintain this stability, improving neuromuscular control and attenuating recurrent LBP $[4,7,13-16]$.

Stabilization exercises are essential in order to provide a base for movement of the arms and legs when supporting weight and to protect the medulla and spinal nerves[17]. Exercise stabilization programs (ST) aim to improve strength, resistance, and motor control of the abdominal and lumbar muscles, with emphasis on the transverse abdominis (TA) and multifidus (MT) (deep trunk muscles) $[6,18]$. Paravertebral and abdominal muscles such as the pelvic musculature and the diaphragm are also important targets for exercise [6].

The contraction of the TA and the MT occur normally upon movement in asymptomatic individuals Hebert et al [8,19-21]; however, in those with LBP contraction of these muscles is slow and prolonged $[1,6,8,20,21]$, indicating a potential risk for lessening of vertebral stability along with problems associated with motor control [6] .

In individuals with lumbago, the large MT undergo atrophy ipsilateral to the point of pain $[6,15,22]$, a decrease in size, activation time and amplitude, dysfunction in co-activation with the abdominal musculature [13] and as well as an increase in intramuscular fat $[1,23]$. These changes in muscle characteristics can be corrected through ST [8], with recuperation of the MT being the most rapid [7].

These exercises should begin in a simple form, associating respiration with contraction of the TA and MT [4], evolving to different postures with movement of the members $[4,16,24]$ and finally including functional posstures[4]. 
Some studies have shown that in individuals with LBP, there is also a decrease in the strength and lengthening of the iliopsoas, due to the connection of this muscle with the pelvis and the lumbar spine [25]. As a result of this attachment, the iliopsoas possibly has a stabilizing role in the column [25]. It is thought that tension in this muscle, formed by the union of the psoas major, psoas minor and the iliac [26] acts bilaterally with the insertion, causing an increase in lordosis, whereas weakening of the muscle reduces its size where both these conditions result in pain $[25,27]$.

Furthermore, according to Sajko \& Stuber [28] the iliopsoas is active during the orthostatic position as well as in flexion and extension of the trunk, all of which suggests that this muscle can act as a truncal stabilizer.

Due to the growing number of studies which show that ST is effective for the treatment of lumbago, as well as pointing to the possible role of the iliopsoas muscle in stabilizing the lumbar spine, the current study was designed to compare the effects of stretching and strengthening of the iliopsoas associated with ST, compared to use of ST alone, in the treatment of LBP.

\section{Patient and Methods}

A randomized clinical study involving three intervention groups was carried out under research project number 377/09, approved by the Research Ethics Committee of the institution.

Sixteen patients were enrolled on the study (two men and 14 women), all with complaints of LBP. Subject age ranged from 22 to 40 years (average $31.27 \pm 6.72$ years) and all participants fulfilled the inclusion and exclusion criteria of the study. Of the initial group, 14 patients (87.5\%) completed the study protocol. One individual withdrew due to health problems, and another did not complete the treatment (Figure 1). The following inclusion criteria were applied: age between 20 and 40 years, a clinical picture and diagnosis of non-specific LBP with a history of more than three months, associated with radiculopathy or otherwise. Exclusion criteria included the following conditions: diagnosis of neuromuscular pathology, either systemic or neurologic; structural deformities; cognitive deficits; previous surgery on the vertebral column or pelvis; history of fracture of the vertebral column, pelvis or hip during the last 12 months; and not perform other treatments for the proposed protocol. All patients were seen at the Department of Orthopedics and Trauma at the Irmandade Santa Casa de Misericórdia de São Paulo Hospital. The study was carried out within the Department of Physiotherapy of the same institution.

After the individuals had been selected for the study and signed a consent form, they were submitted to evaluation of personal details, pain intensity on a scale of one to ten using the Visual Analog Scale (VAS), with 0 representing no pain and 10 the most severe pain imaginable by the patient. The Oswestry Questionnaire for lumbar pain was also applied to assess degree of function. This test is scored $0-50$ points, with 0 representing the best and 50 the worst possible function. Iliopsoas flexibility was measured using a flexometer, and strength with a manual isometric dynamometer. This was tested by the same previously trained examiner having an index of correlation of confidence> 0.71 , who was blinded as to the group being evaluated. After evaluation, the subject was assigned a treatment group using a series of numbers for each group drawn by the study participants from an opaque envelope.

Muscular force evaluation was measured with the patient seated on the evaluation table, with knees and hips flexed at $90^{\circ}$, and hands crossed against the shoulders. The dynamometer was positioned $5 \mathrm{~cm}$ above the femoral condyles and the patient was instructed to exert maximum force in flexion of the hip, while the examiner maintained the dynamometer in the initial position, thus impeding articular motion. This test was repeated three times for each hip, and the average of these values was considered to be the maximum force for the evaluated muscle.

To measure lumbar spine flexibility, the flexometer was positioned in the inframammary region of the trunk. The patient was asked to perform maximal flexion of the trunk in the standing position, with hands placed in front of the body as if touching the floor. This was followed by a maximal truncal extension, with arms held above the head [18].

The equipment used for the study included a Fleximeter $\AA$, Lafayette $\AA$ (model 01163) with weights that varied from 500 to 3000 grams, stretchers for examination, a back rest, a Swiss exercise ball $55 \mathrm{~cm}$ in diameter, and a proprioceptive disc.

There were three treatment intervention groups. This first group was given only ST (ST group, with 6 patients). The second group of four patients was given ST for the lumbar area along with stretching of the iliopsoas muscle (ST and stretching group) while the third group of four patients was given ST as well as strengthening exercises for the iliopsoas (ST and strengthening group).

Treatment was programmed for six weeks but varied between six and 12 weeks (average 9.7 weeks \pm 2.43 ). Two sessions of approximately 45 minutes each were performed with evaluations done at the first, sixth and twelfth sessions. When necessary, the program of visits was changed to accommodate national holidays or missed appointments.

Stretching of the iliopsoas was done with the patient in the decubitus dorsal position with legs overhanging the table. The physiotherapist manipulated the patient's opposite hip and knee into maximum extension by securing each with a different hand and exerting passive extension of the hip to an angle sufficient to generate tension in the muscle. A set of three consecutive extensions was performed on each leg, held for 60 seconds each with intervals of 30 seconds between each movement. Stretching was done for 60 seconds, in accordance with the literature which recommends stretching of the muscle for 30-60 seconds [29]. Iliopsoas muscle strengthening was done using a weight equal to $70 \%$ of the voluntary muscular contraction (VMC) tolerated by the patient, as previously measured using the dynamometer. The individual was seated on the table with feet hanging down and with the support of a back rest. This allowed for a $90^{\circ}$ flexion of 
the hips and knees, from which position, flexion of the hip was performed until the maximum active ADM was reached. Three sets of 20 repetitions were done for each leg. In both positions, the sets were interspersed with a 60 -second rest period.

The ST technique is based on contraction of specific muscles (MT and TA) which help stabilize the lumbar spine. Subjects were taught how to contract these muscles by means of verbal commands and tactile stimuli. Once subjects had learned to contract these specific muscles they were given a previously devised exercise protocol developed by the researchers. This included respiratory exercises, active movement of arms and legs, changes in the decubitus position and more specific exercises of increasing difficulty, according to individuals' ability to maintain muscular contraction (Figure 2) until continuous contractions were possible. Individuals were asked to perform this same type of contraction during their daily activities. The protocol also served as a guide for the researchers in cases where subjects proved unable to perform a particular exercise due to lack of preparation, weakness or increased pain. In this situation, the protocol was continued but an alternative therapy was adopted.

Statistical analysis was performed using SPSS (Statistical Package for the Social Sciences) version 16.0, Minitab 15 and Excel (Microsoft Office 2007). The level of significance for $p$ was set at 0.05 . Ninety-five percent (95\%) confidence intervals were used. Friedman's non parametric test was employed for data analysis.

\section{Results}

On Friedman's test, in which the details of the first evaluation were analyzed in relation to the third evaluation across the three groups, a significant improvement was found on the VAS scale for the ST group ( $\mathrm{p}=0.006)$ (Graph 1). A statistically significant improvement in the two intervention groups was also seen with respect to the Oswestry function score (ST group, $\mathrm{p}=0.003$; ST stretching group, $\mathrm{p}=0.039$, as shown in Graph 2).

However, only the ST strengthening group showed a statistically significant improvement with regards to extension flexibility and in right iliopsoas muscle force ( $\mathrm{p}=0.038$ and 0.05 , respectively)(Graphs 3 and 4).

No significant difference in flexibility of lumbar spinal flexion or in left iliopsoas muscle force was found between intervention baseline and end-point among any of the three groups treated.

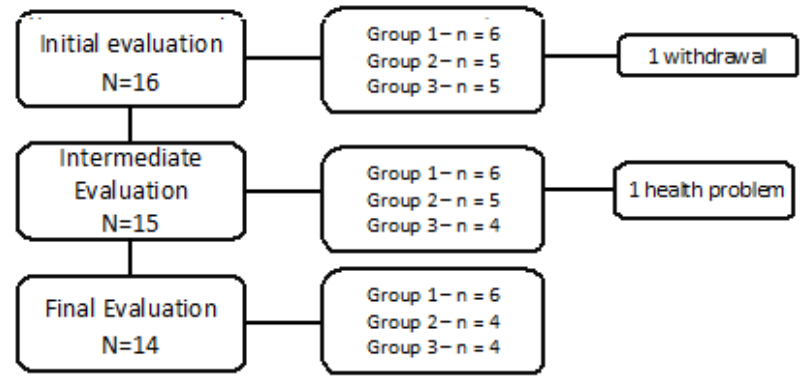

Figure l. Outcome of patients included in the study.

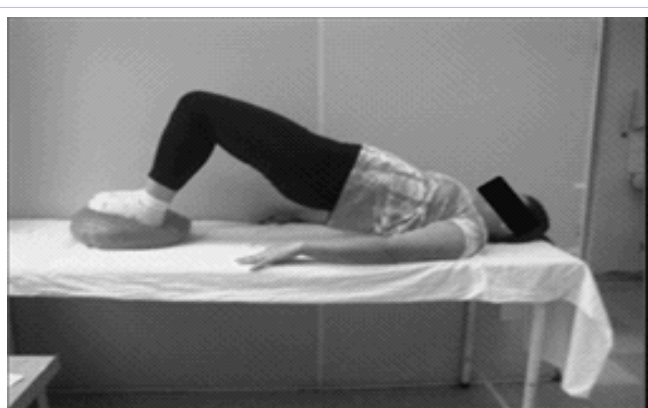

Figure 2.S tabilization exercise with contraction of TA and MI associated with bridge support
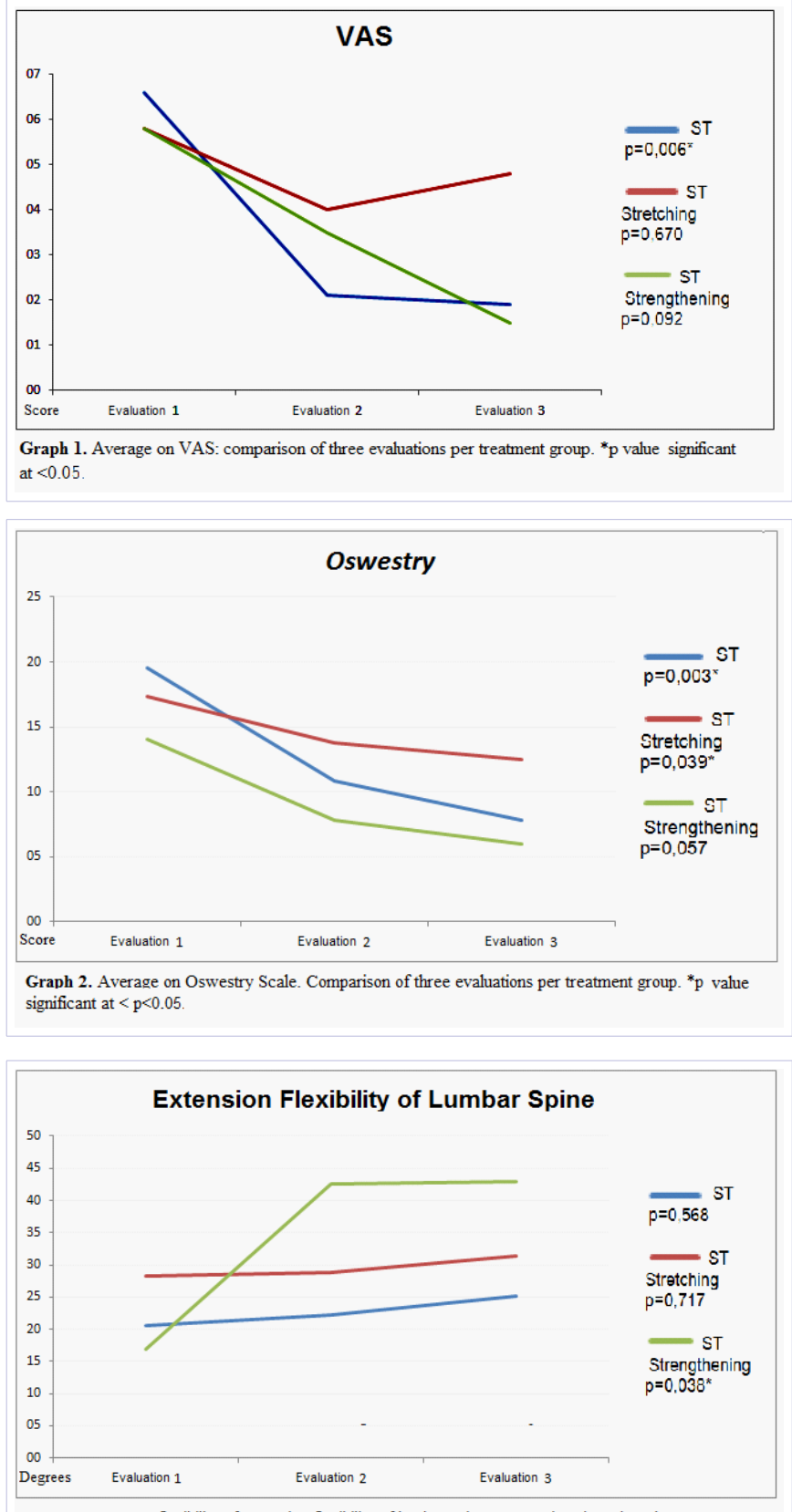

Graph 3. Average flexibility of extension flexibility of lumbar spine. Comparison based on three assessments per treatment group. ${ }^{*} p$ value significant at $p<0.05$. 


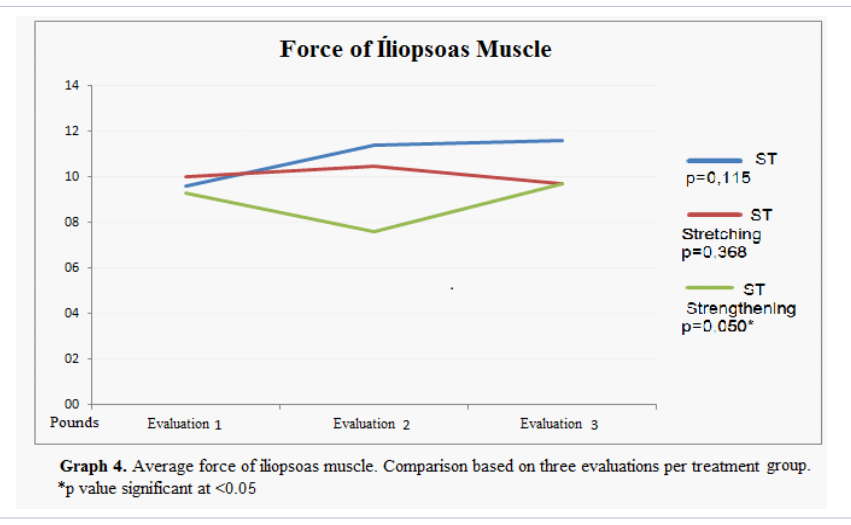

\section{Discussion}

ST has proven effective in lumbago treatment, even when used alone. In addition, combined with other therapeutic techniques, the approach has also been shown to improve chronic LBP [1]. Similarly, the current study affirmed the value of ST treatment when used alone or in combination with iliopsoas muscle strengthening. However, no extra benefit was obtained when ST was associated with stretching of the iliopsoas.

Ferreira et al 2007[3] showed that ST alone improved function in patients with LBP. Our results corroborating this findings, confirming that ST alone was effective for restoring function in patients with lumbago.

Our results also corroborated the findings of the study by [25](Nourbakhsh \& Arab 2002) which ruled out any relationship between the length of the iliopsoas and lumbago, in concluding that lengthening of this muscle by stretching had no effect on pain, columnar flexibility or iliopsoas force.

The same 2002 study [25], which sought to observe the relationship of mechanical factors and LBP by analyzing 600 subjects (300 with lumbago and 300 asymptomatic individuals), confirmed a relationship between muscle force of hip flexors and lumbago, with this force found to be statistically lower in individuals presenting with LBP.

Clinically, iliopsoas stretching has been employed in lumbar spine disorders, justified on the premise that this may increase mobility of the area of the vertebral column [30,31]. These assumptions however were not confirmed in the present study, as patients treated with iliopsoas muscle stretching showed no improvement in columnar flexibility.

Various exercise programs for instability have focused on muscle control of the lumbar spine $[3,32,10]$, with use of isometric contraction of the TA and the MT, and increasing degree of difficulty of the exercises with active movement of the legs [33]. The protocol used in the present study applied these same principles. Standaert et al 2008 [6] suggested the use of verbal instructions, tactile stimuli and specific postures to aid in learning how to do these contractions. The cited authors suggested that a patient was ready to progress to a more difficult level of exercise when able to maintain isometric contraction for 10 seconds without fatigue. The same authors stated that ST typically consisted of six to twelve sessions. These recommendations were followed in the present study.

The main problems encountered in the present study were the difficulties that patients had in adherence to the 12 sessions, prolongation of the time planned for the treatment (which in some cases was double the time anticipated) and failure of one patient to complete all the study sessions. These problems justified the treatment time initially established of six weeks, which is the duration cited by the literature for greatest gain of muscular hypertrophy from strengthening exercises [34]. Nevertheless, we believe that even where increase in iliopsoas muscle strength fails to reach significance, muscular activation would still improve the stabilizing ability of the muscle.

\section{Conclusion}

The results of this study suggest that ST exercises can reduce pain and also improve function and flexibility of the lumbar spine. Stretching of this muscle associated with stabilization appears to be effective only for improving function in individuals with LBP. This suggests that it is preferable to perform ST alone, without the inclusion of iliopsoas stretching exercises.

\section{Limitation}

We can't generalize the data obtained in this study due to the low number of patients included. A larger study is underway to better analysis of the results.

\section{References}

1. Macedo LG, Maher GC, Latimer J, McAuley JH (2009) Motor Control Exercise for Persistent, Nonspecific Low Back Pain: A Systematic Review. Phys Ther 89(1): 9-25.

2. Hayden JA, Tulder MWV, Malmivaara AV, Koes BW (2005) MetaAnalysis: Exercise Therapy for Nonspecific Low Back Pain. Ann Intern Med 142(9): 765-775.

3. Ferreira ML, Ferreira PH, Latimer J, Herbert RD, Hodges PW (2007) Comparison of general exercise, motor control exercise and spinal manipulative therapy for chronic low back pain: A randomized trial. Pain 131(1-2): 31-37.

4. Stevens VK, Coorevits PL, Bouche KG, Mahieu NN, Vanderstraeten GG, Danneels LA (2007) The influence of specific training on trunk muscle recruitment patterns in healthy subjects during stabilization exercises. Man Ther 12(3): 271-279.

5. Hulst M, Vollenbroek-Hutten MM, Rietman JS, Hermens HJ (2010) Lumbar and abdominal muscle activity during walking in subjects with chronic low back pain: Support of the "guarding" hypothesis?. J Electromyography Kinesiol 20(1): 31-38.

6. Standaert CJ, Weinstein SM, Rumpeltes J (2008) Evidence-informed management of chronic low back pain with lumbar stabilization exercises. Spine J 8(1): 114-120.

7. Rackwitz B, Bie R, Limm H, Garnier K, Ewert T, Stucki G (2006) Segmental stabilizing exercises and low back pain. What is the evidence? A systematic review of randomized controlled trials. Clin Rehabil 20(7): 553-567.

8. Hebert JJ, Koppenhaver SL, Magel JS, Fritz JM (2010) The Relationship of Transversus Abdominis and Lumbar Multifidus Activation and Prognostic Factors for Clinical Success With a Stabilization Exercise Program: A Cross-Sectional Study. Arch Phys Med Rehabil 91(1): 78-85 
9. Cowan SM, Schache AG, Brukner P, Bennell KL, Hodges PW, et al. (2004) Delayed Onset of Transversus Abdominus in Long-Standing Groin Pain. Med Sci Sports Exerc 36(12): 2040-2045.

10. Hodges PW, Moseley GL, Gabrielsson A, Gandevia SC (2003) Experimental muscle pain changes feedforward postural responses of the trunk muscles. Exp Brain Res 151(2): 262-271.

11. Renkawitz T, Boluki D, Grifka J (2006) The association of low back pain, neuromuscular imbalance, and trunk extension strength in athletes. Spine J 6(6): 673-683.

12. Goldby LJ, Moore AP, Doust J, Trew ME (2006) A Randomized Controlled Trial Investigating the Efficiency of Musculoskeletal Physiotherapy on Chronic Low Back Disorder. Spine 31(10):1083-1093.

13. Herbert WJ, Heiss DG, Basso DM (2008) Influence of Feedback Schedule in Motor Performance and Learning of a Lumbar Multifidus Muscle Task Using Rehabilitative Ultrasound Imaging: A Randomized Clinical Trial. Phys Ther 88(2): 261-269.

14. Hicks GE, Fritz JM, Delitto A, McGill SM (2005) Preliminary Development of a Clinical Prediction Rule for Determining Which Patients With Low Back Pain Will Respond to a Stabilization Exercise Program. Arch Phys Med Rehabil 86(9): 1753-1762.

15. Hides J, Gilmore C, Stanton W, Bohlscheid E (2008) Multifidus size and symmetry among chronic LBP and healthy asymptomatic subjects. Man Ther 13(1): 43-49.

16. Rydeard R, Leger A, Smith D (2006) Pilates-Based Therapeutic Exercise Effect on Subjects With Nonspecific Chronic Low Back Pain and Functional Disability: A Randomized Controlled Trial. J Orthop Sports Phys Ther 36(7): 472-484.

17. Willardson JM, Fontana FE, Bressel E (2009) Effect of Surface Stability on Core Muscle Activity for Dynamic Resistance Exercises. Int J Sports Physiol Perform 4(1): 97-109.

18. Hides J, Stanton W, Mcmahon S, Sims K, Richardson C (2008) Effect of Stabilization Training on Multifidus Muscle Cross-sectional Area Among Young Elite Cricketers With Low Back Pain. J Hodges Orthop Sports Phys Ther 38(3): 101-108.

19. Ferreira PH, Ferreira ML, Hodges PW (2004) Changes in Recruitment of the Abdominal Muscles in People With Low Back Pain. Spine 29(22): 2560-2566.

20. Allison GT, Morris SL, Lay B (2008) Feedforward Responses of Transversus Abdominis Are Directionally Specific and Act Asymmetrically: Implications for Core Stability Theories. J Orthop Sports Phys Ther 38(5): 228-237.

21. Tsao H, Hodges PW (2007) Immediate changes in feedforward postural adjustments following voluntary motor training. Exp Brain Res 181(4): 537-546.

22. Pressler JF, Heiss DG, Buford JA, Chidley JV (2006) Between-Day Repeatability and Symmetry of Multifidus Cross-Sectional Area Measured Using Ultrasound Imaging. J Orthop Sports Phys Ther 36(1): 10-8.

23. Van K, Hides JA, Richardson CA (2006) The Use of Real-Time Ultrasound Imaging for Biofeedback of Lumbar Multifidus Muscle Contraction in Healthy Subjects. J Orthop Sports Phys Ther 36(12): 920-925.

24. McGill SM, Karpowicz A (2009) Exercises for Spine Stabilization: Motion/ Motor Patterns, Stability Progressions, and Clinical Technique. Arch Phys Med Rehabil 90(1): 118-126.

25. Nourbakhsh MR, Arab AM (2002) Relationship Between Mechanical Factors and Incidence of Low Back Pain. J Orthop Sports Phys Ther 32(9): $447-460$.

26. Penning L (2000) Psoas muscle and lumbar spine stability: a concept uniting existing controversies - Critical review and hypothesis. Eur Spine Journal 9(6): 577-585

27. Kapandji AI. Fisiologia Articular. Vol. 2. 5a ed. Guanabara Koogan, Rio de Janeiro: 2000.

28. Sajko S, Stuber K (2009) Psoas Major: a case report and review of its anatomy, biomechanics, and clinical implications. JCCA 53(4): 311-318.

29. Rosário JLP, Souza A, Cabral CMN, João SMA, Marques AP (2008) Reeducação postural global e alongamento estático segmentar na melhora da flexibilidade, força muscular e amplitude de movimento: um estudo comparativo. Fisioterapia e Pesquisa 15(1): 12-18.

30. Barker KL, Shamley DR, Jackson D (2004) Changes in the cross-sectional area of multifidus and psoas in patients with unilateral back pain: the relationship to pain and disability. Spine 29(22): 515-519.

31. Jorgensson A (1993) The iliopsoas muscle and the lumbar spine. Aust J Phys 39(1): 125-132.

32. Hagins M, Adler K, Cash M, Daugherty J, Mitrani G (1999) Effects of practice on the ability to perform lumbar stabilization exercises. J Orthop Sports Phys Ther 29(9): 546-555.

33. Richardson CA, Jull GA (1995) Muscle control-pain control: what exercise would you prescribe?. Man Ther 1(1): 1-10.

34. Mcardle WD, Katch FI, Katch VL. Fisiologia do Exercício: energia, nutrição e desempenho humano. 6a ed. Guanabara Koogan, Rio de Janeiro: 2008.

35. Hodges PW (2003) Core stability exercise in chronic low back pain. Orthop Clin North Am 34(2): 245-254 